\title{
Slavko Osterc und Karl Reiner im Briefwechsel
}

\section{Slavko Osterc and Karl Reiner in a correspondence}

Matjaž Barbo / matjaz.barbo@ff.uni-lj.si

Department of Musicology, Faculty of Arts, University of Ljubljana, SI

\begin{abstract}
Several hundred different letters and postcards to the Osterc testify his extensive network of national and international contacts. In his entire legacy the correspondence with Reiner appears next to Hába as the most intense and rich. 39 letters have been received from Reiner's side, though the counter-correspondence unfortunately was not found. Reiner's letters to Osterc are full of various discussions about joint projects, the preparation of concerts, changes in interpreters, program etc. Their correspondence was most intense in the years 1933-1935.
\end{abstract}

\section{Keywords}

Slavko Osterc, Karl Reiner, musical correspondence, aesthetics of music, modernism in music 
Slavko Osterc wird als eine von den Zentralfiguren der slowenischen Musik zwischen beiden Weltkriegen betrachtet. Die Bibliographie verschiedener Aufzeichnungen über ihn und sein Werk umfasst eine ganze Reihe unterschiedlicher Wissenschafts-, Fach-, und volkstümlichen Abhandlungen, Beiträge in Enzyklopädien und Lexika, Zeitschriften, Memoirenliteratur usw. Anlässlich des 100-jährigen Geburtstags des Komponisten wurden sogar zwei Symposien veranstaltet und zwei Fachfestschriften veröffentlicht (Muzikološki zbornik 1995, Kuret 1996). Parallel damit erfolgte eine umfangreiche Ausstellung mit dem Katalog, worin eine genaue Beschreibung der wichtigsten Lebensstationen des Komponisten, sowie Aufzeichnung seiner Werke und Erstaufführungen davon enthalten sind (Loparnik 1995). Darüber hinaus sind dem Komponisten und seinem Werk bedeutende Monografien des Danilo Pokorn (1969), Andrej Rijavec (1972), Dragotin Cvetko (1988, 1993) und Katarina Bedina (1995) gewidmet. Es gilt hervorzuheben, dass einige Forschungsarbeiten nicht zuletzt aus den Federn der ausländischen Fachleute stammen, darunter befinden sich auch tschechische Musikologen. Davon hebt sich die eingehende und bedeutende Analyse des Briefwechsels zwischen Osterc und Hába durch Vlasta Reittererová und Hubert Reitterer (2005) ab.

Slavko Osterc wurde 1895 in Veržej am Fluss Mura, einem Bauern- und Marktfleckenumfeld geboren, woraus die ihm eigene verschmitzte Schalkhaftigkeit und spöttische Lust auf Herausforderung von der typischen unsentimentalen Derbheit durchtränkt wurde (Šiftar 1963, Tibaut 1995).

Nach dem Realgymnasium besuchte er das Lehrerbildungsseminar in Maribor (1909-1914). Das Musikleben in Maribor war nicht so aufregend wie es sich in anderen größeren Städten des österreichischen Kaisertums gestaltete, jedoch war damals der junge Osterc insbesondere vom deutschen Theater völlig hingerissen.

In Maribor nahm er auch Kontakt mit ausgezeichneten Musikern auf. Darunter waren insbesondere Hinko Druzovič (1873-1959) und Emerik Beran (1868-1940) von Bedeutung. Beran war ein talentierter tschechischer Musiker, stammend aus der berühmten Brno Orgelschule des Leoš Janáček (Tyrell 2011, Weiss 2008). Sein Unterricht wurde vom hohen Niveau der Kenntnisse sowie Enthusiasmus geprägt, womit er auch Osterc begeisterte. Beran machte Osterc offenkundig Mut bei anfänglichen Kompositionsversuchen und stärkte dessen kreatives Selbstbewusstsein.

Nach beendeter Ausbildung trat Osterc eine Lehrersstelle an und erfuhr ganz bald auch die ersten freundlich gesinnten Kritiken der Aufführungen seiner Werke (gemeinsam mit dem ersten Werk größeren Umfangs, der symphonischen Dichtung Krst pri Savici - Die Taufe bei Savica).

Osterc war sich seiner spärlichen Kompositionskenntnisse bewusst, gleichzeitig aber auch überzeugt, dass es auf dem slowenischen Gebiet weder eine Persönlichkeit noch Schule gibt, die ihm die Fachhorizonte zufriedenstellend öffnen könnten. Womöglich war Beran für die Wahl des Studienortes von entscheidender Bedeutung, vielleicht wurde sie aber vom nationalen Bewusstsein beeinflusst, worin etwas vom antideutschen Trotz und echten slawischen Empfinden steckte. Jedenfalls entschied er sich für Prag, berühmt nach aufregendem Musikleben, reicher Musiktradition und hochgeschätztem Musikkonservatorium. 
Das Bildungsministerium genehmigte seinen Antrag auf Weiterbildung, womit er im Herbst des Jahres 1925, bereits 30 Jahre alt, begann. Aufgrund der vorgelegten Musikstücke wurde er ohne Prüfung im dritten Jahrgang des Landeskonservatoriums in Prag angenommen. Osterc hörte bei Karel Boleslav Jirák Kompositionstechnik und Instrumentierung, Musikformen lernte er bei Jaroslav Křička, Dirigieren wurde von Otakar Ostrčil und dem ehemaligen Dirigenten in Ljubljana Václav Talich, und Ästhetik von Václav Štěpán unterrichtet. Bei der Immatrikulierung wurde er dem Komponisten Vitěslav Novák zugeteilt, doch es scheint, als ob sie miteinander nicht harmonierten, so dass er nach dem ersten Semester Schüler von Jírak (1891-1972) wurde. Obwohl dieser nur etwas älter als Osterc war, stellte er die entsprechende Lehrerautorität auch als einer der ausgeprägten Befürworter der modernistischen Strömungen dar.

Es scheint, dass das vorher intensive Komponieren des Osterc durch Studienpflichten etwas gehemmt wurde. In der Prager Zeit besuchte Osterc regelmäßig Konzerte und Opernaufführungen, da er offenkundig beabsichtigte, das Prager Kulturleben voll zu genießen. Er schrieb seine Eindrücke auch sorgfältig nieder. In seinem Nachlass ${ }^{1}$ ist ein äußerst interessantes Heft mit näherer Beschreibung von Konzerten und Vorstellungen, denen er in Prag beiwohnte, erhalten. Darin werden Musiker, Solisten, Dirigenten und Orchester, ziemlich näher das Konzertprogramm und kurz auch seine Bewertung der Aufführung vermerkt. Aus der Beschreibung geht hervor, dass er wöchentlich im Durchschnitt gar einige Veranstaltungen besuchte. ${ }^{2}$

Bereits im März 1926 fand in Prag unter der Schirmherrschaft der Slowenischen Schülergenossenschaft (Slovenska dijaška zadruga), vermutlich eben unter Osterc, ein Konzert der slowenischen zeitgenössischen Musik statt. Im Konzert erklungen die Werke von E. Adamič, A. Lajovic, J. Ravnik, R. Savin, L. M. Škerjanc und natürlich Osterc. Dabei hob der Kritiker in der tschechischen Zeitschrift Narodni Osvobózeni (6. 3. 1926) hervor, „dass lediglich Osterc einen erkennbaren eigenen Klang hat" (Cvetko 1993, S. 37). Das Prager Radiojournal veranstaltete in diesem Jahr (1926) noch einen "jihoslovansky“ Abend, wo unter anderem auch die Musik von Savin sowie Nokturno und Štiri belokranjske (Vier Stücke aus Bela Krajina) aufgeführt wurden (Cvetko 1993, S. 38). In Prag erschienen im selben Jahr (1926) auch Štiri belokranjske für Stimme und Klavier, das erste im Ausland veröffentlichte Werk des Komponisten.

Im Herbst des Jahres 1926 immatrikulierte sich Osterc nebst viertem Jahrgang des Prager Konservatoriums als erster Ausländer auch am berühmten Kurs der Viertelkomposition, geführt von Hába. Nach einem Jahr (die Bestätigung trägt das Datum des 21. Juni des Jahres 1927) absolvierte er ihn mit dem Preludij za klavir (Präludium für Klavier). Zweifellos wurde unter dem Hábas Einfluss die modernistische Ästhetik von Osterc in den Prager Jahren noch verschärft.

1 Slavko Osterc Nachlass, Mappe Kronika, Musiksammlung der National- und Universitätsblibliothek, Ljubljana.

2 Unter den Komponisten, deren Werke er in Prag kennen lernte, wurde er besonders geprägt von Stravinsky mit seinem Le sacre du printemps, dann Bartók mit Dem wunderbaren Mandarin, Hindemith und seinem Konzert für Orchester und der Oper Cardillac, enttäuscht war er aber von der Křenek Oper Jonny spielt auf. 
Im Jahre 1927 absolvierte Osterc das Studium am Prager Konservatorium. Im Juni wurden im Abschlusskonzert der Konservatorium-Absolventen Štiri karikature (Vier Karikaturen) für Piccolo, Klarinette und Fagott, Osem anekdot Charlia Chaplina (Acht Charlie Chaplin Anekdoten) für Stimme und 11 Instrumente sowie Prvi godalni kvartet (Das erste Streichquartett) des Osterc aufgeführt. Osterc erfuhr wiederholt positive Kritiken, er soll sich »in der Richtung der französischen Moderne bewegen, mit seinen Werken überraschen und die Technik des Komponierens beherrschen«(Cvetko 1993, S. 38). In der Zeitschrift Československá republika wurde sein Werk auch von Hába positiv bewertet, indem er schrieb, dass Osterc „der meist talentierte Künstler unter dem jugoslawischen Nachwuchs ist" (Cvetko 1993, S. 38). Er betonte dabei, Osterc beherrsche den modernen Klang und Polyphonie, obwohl er diese auf seine eigene Art gestalte. Er hob den Humorsinn des Komponisten und sein Gefühl für Rhythmus hervor und prophezeite ihm eine bedeutende Entwicklung.

Osterc wollte sein Studium in Prag noch verlängern, jedoch konnte er die entsprechende Genehmigung des Bildungsministeriums nicht mehr einholen. So begann er im September 1927 am Landeskonservatorium in Ljubljana Harmonie, Kontrapunkt, Komposition, Instrumentierung, Formlehre mit Analyse, Ästhetik und Musikgeschichte zu unterrichten.

Darüber hinaus nahm er am breiteren Kulturleben intensiv teil. Unter anderem wurde er bei der Gründung der Musikzeitschrift Nova muzika im Jahre 1928 Redakteur für den Notenteil. Bereits im ersten Jahrgang veröffentlichte er die Werke zahlreicher tschechischer Autoren, unter ihnen K. B. Jirák, O. Zich, V. Novák, J. Suk, J. B. Foerster, O. Ostričil, E. Axman, B. Vomáčka, J. Křička, Rudolf Karel und A. Hába mit einem Vierteltonmusikstück.

Vor allem begann aber Osterc immer ausdrücklicher ein breites internationales Netz von Freunden zu knüpfen. Im Jahre 1931 ging er erneut nach Prag, wo im Rahmen von Spolek pro moderni hudbu im Konzert mit Vierteltonmusik (7. 6.) Karel Reiner, damals noch ein Student von Hába (in der Zeitabschnitt 1929-1932), das Preludij za klavir von Osterc aufgeführt hatte, womit ihre Bekanntschaft dokumentiert begann.

Im Mai des folgenden Jahres (1932) nahm Hába auf Einladung des Osterc an den Ersten slowenischen Musikfestspielen in Ljubljana teil (Loparnik 1995, S. 24). Für die Festspiele stellte Hába auch eine Vorlesung über die Vierteltononmusik bereit.

Hába war damals der IGNM-Sekretär. Bei dieser Gelegenheit ermunterte Hába Osterc zur Neuerrichtung der jugoslawischen Sektion, deren Tätigkeit ein wenig ins Stocken geraten war. ${ }^{3}$ Außerdem nahm damals Osterc durch Hába Kontakte mit Universal-Edition an.

Obwohl hochgeschätzt als Komponist, war Osterc mit seinen scharfen modernistischen Grundsätzen und persönlicher Nichtbereitschaft zu Kompromissen des Öfteren ein Dorn im Auge für die allgemeine öffentliche Meinung und mit bestimmten Musikkreisen zerstritten. Vermutlich war das auch Grund dafür, dass Osterc bei der Gründung der

3 Bereits im Juni 1932 nahm Osterc zusammen mit M. Bravničar sowie J. Slavenski und M. Milojević aus Belgrad an den zehnten ISCM - Festspielen in Wien teil. In den Besprechungen mit A. Hába und Edward J. Dent, dem Vereinsvorsitzenden bereits seit Gründung davon im Jahre 1922, wurde damals beschlossen, die jugoslawische Sektion neu zu errichten. Osterc wurde zum Sekretär gewählt. 
neuen Musikakademie in Ljubljana im Jahre 1939 keine Stelle daran bekam, obwohl er diese selbstverständlich erwartete. Die Enttäuschung wurde noch von einer fortschreitenden Krankheit begleitet, wodurch nicht nur sein pädagogischer Eifer, sondern auch die Lebenskräfte schwanden. Unmittelbar nach Beginn des Krieges in Jugoslawien starb er im Mai 1941.

Innerhalb der letzten Lebensjahre des Osterc schrumpfte sein ehemals so breiter Freundeskreis, zu dem seine Schüler und andere Musiker sowie einige prominente Intellektuellen gehörten, so zum Beispiel der Literaturhistoriker und Slowenist Ivan Prijatelj und der Schriftsteller und Dramatiker Fran Govekar. Einige von diesen Freunden verbanden sich in der informellen Gruppe „Die alten Slawen“ (Stari Slovani), wozu nach Osterc auch einige ausländische Musiker gehörten, unter ihnen der serbische Komponist Miloje Milojević sowie A. Hába und K. Reiner. Mitunter trafen sie sich im dem bekannten Gasthaus von Ljubljana „Pri Krčonu“. Sonst „amtierte“ Osterc selbst regelmäßig den ganzen Tag auch im Kaffeehaus „Zvezda“, oder ab und zu in „Pri Figovcu“, wo er unter anderen seine Post in Empfang nahm.

Osterc konnte mit seiner modernistischen Ästhetik und gleichzeitiger persönlicher Anziehungskraft seine Schüler ansprechen und sie mit Widerstand gegen den sentimentalen Romantizismus und der Befürwortung des Neuen zu prägen. Weil sein Unterricht, wovon die Memoiren seiner Schüler Zeugnis ablegen, nicht streng systematisch und ausschließend war, hat er ein buntes Bild von mancherlei unterschiedlichen Autoren mit eigenständigen Kunstphysiognomien und ästhetischen Ansichten erzogen (Loparnik, 1984). Womöglich wurden sie vor allem dadurch verbunden, dass sie sich der Tatsache rühmten, Schüler des Osterc und als solche Verteidiger des Neuen in der Musik zu sein.

Vermutlich wurde dieser pädagogische Ansatz in Prag angenommen, da ähnliche Züge auch die Hába „Schule“ definieren, die als Ergebnis der historischen und ästhetischen Interpretation von Spurný problematisiert wird (2011, S. 146). So ist für beide Lehrer Mangel an System bezeichnend (Spurný 2011, S. 141), ausgehend von der so genannten Musik der Freiheit (wie sie Hába selbst nannte). Und wenn Hába bei seinem Unterricht Mikrotonalität und Athematizismus hervorhob (Spurný 2011, S. 141), ist beides betont auch bei Osterc zu treffen, der sich zwar mit der Vierteltonmusik nicht weitgehend befasste, ${ }^{4}$ jedoch die Bedeutung des Athematizismus ausdrücklich hervorhob.

„Er [der Schüler] soll eigenes freies Kombinieren üben, “ schrieb Hába im Jahr 1927 (Spurný 2011, S. 141), ebenfalls könnte der gleiche Satz auch als Motto des Unterrichtens von Osterc gelten.

Karl Reiner schrieb über die Hába Schule vielsagend wie folgt.

4 Bei Osterc findet man nur einige Vierteltonwerke: Tri skladbe za četrttonski klavir (Drei Kompositionen für Vierteltonklavier), 1927, Štiri Heinejeve pesmi (Vier Heine Lieder), 1931 und Cvetoči bezeg (Der blühende Flieder),1936. Darüber hinaus befasste er sich Anfang der 30-en Jahre sogar mit der Schönbergs Dodekafonie: Kvintet za pihala (Bläserquintett), 1932 und Koncert za klavir in pihala (Konzert für Klavier und Bläser), 1933. Bei dem symphonischen Musikstück Ouverture classique (1932) ist in den Skizzen sogar eine Systematisierung harmonischer Spannungen zu empfinden, die an Hindemith erinnern. 
„Das Interessante an Hába Schule ist es, dass es sich um keine einheitliche Schule handelt. Jeder von seinen Schülern ist anders, jeder entwickelt sich in einer anderen Richtung. Sie haben gewählt, was ihnen und ihren Talenten und ihrem Stil, ihrem Sozialumfeld sowie ihrer Nationalität passt. Typisch für diese Schule war sogar eine Entwicklung des Nationalcharakters der Schüler aus verschiedenen Nationen." (Spurný 2011, S. 143).

Ähnliches ist über die Osterc Schule bei Primož Ramovš zu lesen, einem von den prominenten slowenischen Komponisten aus der 2. Hälfte des 20. Jahrhunderts:

„Seine Stunden waren wahrhafte Kreationen. Der Stoff zwar nach dem Schulplan, jedoch frei bearbeitet, wahrscheinlich ein jedes Mal anders. Man konnte nie wissen, was kommt, nichts von dem Gymnasialrattern der immer gleichen Lehren.“ (Loparnik 1984, S. 46)

Womöglich wirkte sich das Konzept der »Musik der Freiheit « auch darauf aus, dass sich auch die Schüler des Osterc mit der Vierteltonmusik fast nicht befassten - ausgenommen einige Versuche von Franc Šturm und Radoslav Hrovatin (Bedina 1981).

Gleichzeitig aber sandte Osterc seine Schuler regelmäßig nach Prag. So wies er bereits den ersten Diplomanten, Pavel Šivic an, zu Suk und Hába zu gehen, später regte er dazu noch Marijan Lipovšek, Demetrij Žebre, Franc Šturm und Dragotin Cvetko an. Wie umgekehrt auch Osterc in Prag als Lehrer geschätzt war, beweisen die Worte von Suk in dem Brief an Osterc vom 23. 6. 1933: „Ihr Wort genügt, dass Herr Šturm [zum Studium] angenommen wird“ (Loparnik 1995, S. 26). ${ }^{5}$

\section{Der Briefwechsel}

Unter den slowenischen Musikern pflegte Osterc zweifellos die weithin meisten Kontakte mit einigen führenden Musikpersönlichkeiten seiner Zeit. Es sind mehrere hundert verschiedene Briefe, Ansichtskarten und Postkarten erhalten, die ein außerordentlich weit ausgebreitetes Netz von nationalen und internationalen Kontakten bezeugen. Diese wurden nach seiner Abreise nach Prag, selbstverständlich aber insbesondere nach seiner Heimkehr, stärker und immer mehr verzweigt. Dabei blieb er im regelmäßigen Briefverkehr mit Prager Professoren J. Křička, J. Suk, K. B. Jirák und insbesondere A. Hába ${ }^{6}$ (einige Briefe von Osterc an Hába und Jirák befinden sich auch im Nationalmuseum Prag - Tschechischen Museum der Musik Národní muzeum Praha - České muzeum hudby Praha).

\footnotetext{
$5 \quad$ Šturm schreibt im Brief an Osterc (15. 11. 1934) zwar: „Suk hat mich in die Meisterschule nach Hábas und Reiners Intervention angenommen. "(Cvetko 1988, S. 338)

6 Die ersten erhaltenen Hábas Briefe datieren etwa aus dem Anfang der 30-en Jahre, danach erfolgt kontinuierlicher Briefwechsel bis zum Tod von Osterc. Es ist interessant, dass sie dabei oft die deutsche Sprache benutzten, der sie beide mächtig waren. Einige Aufzeichnungen sind in der tschechischer und sogar auch in der slowenischen Sprache abgefasst.
} 
Ein Teil des Briefwechsels zwischen Hába und Osterc ist mit der Tätigkeit der Internationalen Gesellschaft für Neue Musik (IGNM) verbunden (Stefanija 1995). Über Kontakte mit den IGNM Mitgliedern suchte Osterc nach Möglichkeiten zur Aufführung seiner Werke sowie der Musikstücke slowenischer und jugoslawischer Autoren im Ausland und allerdings auch für die Präsentation des zeitgenössischen Musikschaffens im Inland. Auf den IGNM-Festspielen wurden somit in den 30-en Jahren regelmäßig auch Werke des Osterc präsentiert. Als im Jahre 1934 in Florenz die Gradnikove pesmi (Gradnik Lieder) für Kontraalt und Streichquartett (präsentiert von Franja Golob-Bernotova) aufgeführt wurden, schrieb Karel Reiner als Kritiker, sie seien im Vergleich zu anderen Musikstücken auf den Festspielen sogar das stärkste Werk gewesen, positive Bewertung als eine von den Gipfelleistungen auf den Festspielen erfuhr er auch vom slowakischen Komponisten Alexander Moyzes. Im folgenden Jahr 1935 führte Reiner in Prag das Koncert za klavir in pihala auf, das bereits ein Jahr vorher im Radiojournal erstaufgeführt wurde (Loparnik 1995, S. 26). Der Komponist selbst reiste nach Prag und nahm erneut einige Kontakte mit den tschechischen Musikern auf. Im Jahre 1936 wurde Osterc nach Paris zur Jury für die Auswahl von Werken für die Aufführung auf den Festspielen eingeladen. Im Jahre 1938 wurde dann in London das Symphoniewerk Movement symphonique aufgeführt, besonders wurde aber Osterc mit der Aufführung von Passacaglia und Koral im Eröffnungskonzert auf Festspielen in Warschau 1939 gewürdigt.

Osterc korrespondierte oft mit ehemaligem Schüler Franc Šturm, insbesondere während dessen Fortbildung in Prag und Paris. Šturm berichtete Osterc über das tschechische Musikleben und Osterc blieb über ihn auch in Kontakt mit den Prager Freunden. Diesbezüglich tritt Karel Reiner hervor, der Prager Pianist und Hábas Mitarbeiter, der ein aufrichtiger Freund von Šturm war, wie es aus dem Briefwechsel zwischen den beiden ersichtlich ist.

Bereits im ersten Brief an Osterc bezeichnete ihn Reiner wie folgt: „euer Šturm ist ein lieber Junge; wir werden ihn schon tüchtig bearbeiten “ (12. 10. 1933; Cvetko 1988, S. 285).

Im gesamten Nachlass von Osterc erscheint neben dem Briefwechsel mit Hába gerade der Briefwechsel mit Reiner als am meisten intensiv und reich. Darin sind 39 Briefe von Reiners Seite erhalten, verwahrt in der National- und Universitätsbibliothek von Ljubljana, währenddessen die Gegenkorrespondenz nicht gefunden wurde. Der erste erhaltene Reiners Brief an Osterc ist auf den 12. 10. 1933 datiert, obwohl sie sich zweifellos bereits vorher gekannt haben - weniger wahrscheinlich aber in der Zeit des Studiums von Osterc in Prag, da dieses von Osterc im Jahre 1927 absolviert wurde und Reiner 1929 damit begann, jedoch gewiss wenigstens seit 1931, seit der bereits erwähnten Reiners Erstaufführung des Viertelton-Präludiums (7. 6.) in Prag. Reiner, der praktisch in ganz Europa auftrat, führte in seinen Konzerten von moderner Musik auch danach regelmäßig die Werke von Osterc auf. Darunter spielte er oft Toccata und Arabeske, temperamentvolle Musikstücke, die auch andere ausländische Pianisten in ihren Konzerten präsentierten.

Reiners Briefe an Osterc sind voll von verschiedenen Besprechungen über gemeinsame Projekte, Konzerte mit slowenischer und tschechischer Musik, Auftritte im Radio, im Rahmen von Př́ítomnost, IGNM-Festspielen, in Ljubljana, Prag usw. Darin folgt man den Vorbereitungen von Konzerten, Verlegungen davon, Änderungen in Interpreten, 
Programm u. ä. Reiner regte Osterc auch konstant zum Schreiben an. So schreibt Reiner 1933: „ihr klavierkonzert möchte ich sie bitten, sofort nach vollendung hierher zu schicken, damit ich es noch heuer in radiojournal spielen kann. " (2. 11. 1933, Cvetko 1988, S. 286)

Oder: „ein 'vierteltöniges' klavierwerk von ihnen brauche ich natürlich auf jedenfall. “ (2. 11. 1933, Cvetko 1988, S. 286).

Ständig wurden Konzerte geplant, wie zum Beispiel im Jahre 1934 in Ljubljana:

„programm: selbstverständlich: osterc, klavierkonzert, von euern leuten eventuell šturm oder wen ihr sonst noch vorschlagt, von unseren: hába, srnka, zavadi, střižek, krejčí, ježek usw. genaues programm eines kammermusikabends hat ja zeit in florenz zusammenzustellen." (19. 3. 1934, Cvetko 1988, S. 287)

\section{Oder in Prag:}

„Wir veranstalten dafür im November oder Oktober in der Přitomnost noch einen Abend der Laibacher Kompositionsschule Slavko Osterc. Ist berets. fix!!“ (27. 3. 1934, Cvetko 1988, S. 287)

Reiner ist bereit, daran besonders intensiv zu arbeiten, wie er aus Rom schreibt:

„Werde natürlich für dieses Monsterkonzert wahnsinnig üben müssen. Wird schon auf 5 Stunden täglich herauskommen. Dafür garantiert gut, hoffentlich.“ (25. 4. 1934, Cvetko 1988, S. 288)

In den Briefen findet sich unter anderem auch die schon lange angekündigte Erstaufführung des Koncert za klavir in pihala von Osterc, die am 13. 3. 1934 mit dem Dirigenten O. Jeremiáš und dem Orchester des Prager Radiojournals stattfand. Die Aufführung wurde erfolgreich wiederholt, wie bereits erwähnt, später noch auf den IGNM-Festspielen. Bereits einen Tag nach dem Konzert, am 14. 3., schreibt Reiner an Osterc:

„Wenn man bedenkt, dass wir für das Konzert insgesamt $2 \frac{1}{2}$ Stunden Proben hatten, dass ich selbst es knapp 14 Tage üben konnte, dass wir am Abend um 6h letzte Probe hatten, ich von 8-10 Uhr noch im osvobozené bei Ježek Schlager spielte, ist die Aufführung noch sehr gut abgelaufen. Und vor allem: es hat allen Anwesenden und was ich so bis Mittag hören konnte, auch sonst ganz ausgezeichnet gefallen, alle rühmen die frische Musikalität, die gelungenen Modulationen und hie und da hört man endlich auch, dass erst jetzt zu sehen ist, um wieviel armer so ein unsriger Bořkovec oder Hindemith ist. Auf jedenfall gratuliere ich. [...] und noch etwas, damit Sie nicht zu stolz werden und ich eine ersehnte Gelegenheit zum Schimpfen habe: bitte, den Solopart immer als 2 systemigen Klavierauszug schreiben! Es ist für den Solisten sehr unangenehm, Pausen zählen zu müssen und nicht zu wissen, was dort vorgeht. Die Partitur hatte ich nämlich gerade 2 Stunden. In denen zeichnete ich mir ein, wann und bis wohin ich solo spiele. So, jetzt ist mir leichter.“ (Cvetko 1988, S. 286-287)

Auch sonst wurden die Werke von Osterc im Radiojournal oft aufgeführt. Bereits 1931 wurde im Konzert der Tschechischen Philharmonie, geführt von Jirák und übertragen 
von Radiojournal Praha und jugoslawischen Rundfunksendern, das Koncert za violino in sedem instrumentov (Konzert für Violine und sieben Instrumente) erstaufgeführt. ${ }^{7}$ Avgust Šuligoj mit dem damals berühmten slowenischen Jugendchor Trboveljski slavčki führte das Quartett in fast zwanzig Konzerten während der Tournee durch die Tschechoslowakei auf, unter anderem auch in Prag und im Prager Radiojournal (und auf dem Weg nach Hause noch im Wiener Musikverein).

Im Jahre 1936 erfolgte im Radiojournal die Erstaufführung des Kvintet za pihala, präsentiert von dem Prager Bläserquintett. Im danach folgenden Jahr führte das Ondřičekquartett (beziehungsweise das Radioquartett im Prager Radiojournal noch das Drugi godalni kvartet (Das zweite Streichquartett) erstmals auf.

Reiner schätzte Osterc sehr hoch, seiner Ansicht nach war er der beste Komponist im slawischen Süden überhaupt:

„Habe unlängst mit Suk gesprochen. Trotz verschiedener Einstände bist Du der beste Jugoslawische Komponist. Das wissen wir aber schon längst.“ (19. 11. 1934, Cvetko 1988, S. 293)

Bei der Vorbereitung des jugoslawischen Konzertes in Prag 1935 schreibt er, es soll die Klaviertoccata auf dem Programm sein:

„Abgesehen davon konnte ich Dich nicht mit einem zu grossen Werk vertreten, weil du heuer hier der meist gespielteste jugoslawische Autor bist und ich persönlich den radikalsten Osterc präsentieren will.“ (8. 3. 1935, Cvetko 1988, S. 296)

Ihre Freundschaft wurde nicht einmal durch die manchmal offenen Meinungsverschiedenheiten ästhetischer Natur zwischen den beiden getrübt. So findet man unter anderem die Kritik an Šturm: „Sturms merkmale waren gerade die, keine merkmale zu haben: absolut keine kräftige melodik (lange würste) [eine Wurst war das Synonym für Musikstück in der Kommunikation von Osterc mit seinen Studenten und offensichtlich auch mit Reiner] - rythmus gleichförmig, ohne abwechslung. “(4. 7. 1934, Cvetko 1988, S. 291). Daneben ist offen Kritik auch an Osterc in einem von den Briefen an ihn:

„[...] in deinen kompositionen ist vielleicht für dich die dynamik überflüssig. objektiv aber fehlt sie, nur ist bei dir das natürliche temperament so stark, dass man auch ohne dynamik weiterkommt. zugeben aber wirst du auch müssen, dass auch deine Sachen grossenteils unlyrisch sind. aber auch lyrik ist notwendig. sangbare melodik.“ (4. 7. 1934, Cvetko 1988, S. 292)

Reiner stellte später auch andere Osterc Schüler unter Kritik:

„Cvetko 'arbeitet' noch mit neuen Klängen, von Sturm gefällt mir das erste Lied, das übrige noch nichts besonders. Sehr interessieren möchte mich, ob die Burschen diese komplizierten Tatwechsel nur so oder aus der Notwendigkeit des Slowenischen oder Volkscharakter schrei-

7 Jirák wird 1930 Leiter des Musikprogramms und der erste Dirigent des Radiojournals, was vielleicht auch der Grund ist, warum Osterc mit ihm intensiver korrespondiert. 
ben. [...] Mich interessiert es besonders, weil ich auf Komplizierung des Rhythmus innerhalb des Taktes, (bis zu Polyrytmik) gehe, die Burschen aber komplizierte Taktarten miteinander mischen. Für mein Gefühl fallen die Perioden etwas auseinander." (25. 12. 1936, Cvetko 1988, S. 300)

Diesbezüglich kommentiert Cvetko diesen Teil des Briefwechsels nur karg, Reiner habe „kritisch über die Kompositionspraxis einiger slowenischen modern orientierten Autoren gesprochen, insbesondere in Bezug auf Taktwechsel und komplizierte Rhythmen "(Cvetko 1988, S. 109).

Reiner war manchmal auch kritisch gegen andere eingestellt. In einem Brief ärgert er sich über den serbischen Komponisten, der ihm aus Belgrad nicht geantwortet habe:

„das schwein hat alle meine orchestersachen (existieren nur einmal und gehören dem Radio), antwortet überhaupt nicht, ich werde die sachen irgendwie gerichtlich oder über unsere gesandtschaft herausbekommen müssen.“ (9. 1. 1935, Cvetko 1988, S. 294)

Osterc und Reiner trafen sich gewiss auch persönlich auf regelmäßiger Basis, ihre Kontakte gingen in eine echte Freundschaft über. Das wird durch zahlreiche Briefe nachgewiesen, in denen Reiner vom anfänglichen „Sie“ im Jahre 1934, nach einem längeren Aufenthalt in Slowenien anlässlich seines Konzertes im Mai desselben Jahres, zu „Du“ übergeht. Zu der Zeit wurde die Freundschaft zwischen ihm und Osterc offenkundig enger, was nicht zuletzt aus den herzlichen gemeinsamen Fotos hervorgeht.

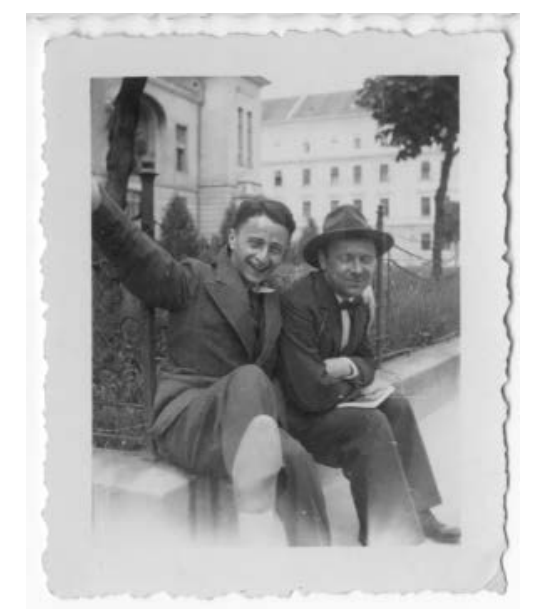

Abb. 1 S. Osterc mit K. Reiner anlässlich seines Besuches in Ljubljana im Mai 1934. Über das Foto schreibt Šturm an Osterc: "Ich war bei Reiner und sah die Bilder aus Ljubljana. Überaus gut ist jene vor dem Dramagebäude. (Du und Reiner)" S. 338); Musiksammlung NUK in Ljubljana, nach Genehmigung.

Er adressiert Osterc nunmehr mit unterschiedlichen freundlichen und geistreichen Kosenamen: „lieber alter Diktator“, „Lieber alter Slovane“, „Lieber Slavko“, „Lieber alter 
slovanischer Diktator“, „Lieber stari slovinec“, auch „Mily stary Slovane“ u. ä. Er selbst unterzeichnet in verschiedensten Weisen wie „Dein Karlik“, auch „Dein Reiner“, „Dein jüdischer Slovane“, „dein Kadett“ oder „Dein junger Slovane Ahojsky“. Nach der Aufführung der Toccata und Arabeske im Jahre 1935 unterzeichnet er den Brief als „Dein tolkater arabeskeur“ (27. 3. 1935, Cvetko 1988, S. 297)

Bereits in einem von den ersten Briefen, als er ankündigt, dass sie sich in Florenz treffen würden, sagt Reiner: „Ich freue mich schon sehr auf Eure Bande.“ (14. 3. 1934, Cvetko 1988, S. 286)

Auch Šturm andererseits schreibt an Reiner darüber, dass er sich auf das gemeinsame Zusammensein im bekannten Gasthaus von Ljubljana freut: „Ich freue mich schon näheres über alles zu erfahren, besonders über den 'Krčon' sowie auch über die Krčon-gemeinde. "(Cvetko 1988, S. 338)

Dass ihr Zusammensein oft recht munter vor sich ging, zeigt auch das direkte Brief von Reiner an Osterc nach dem Gastspiel (und Trinkgelagen) im Mai 1934 in Ljubljana, als er nachfolgend ein Konzert in Venedig zu geben hatte, er habe es mit „Caffé espresso, byrrha usw. recht gut durchgebracht und mit ungefähr 5 Katzen am canalle grande ein wunderbares Konzert veranstaltet. “ (13. 5. 1934, Cvetko 1988, S. 289)

Einer von den Briefen aus 1934 endet mit freundlichem Gruß:

„Lieber alter Diktator! Ich schliesse heute meinen Brief und danke Dir nochmals herzlichst für Deine staro slovanská Gastfreundschaft. Grüße Deine Frau und die kleine und ganz Ljubljana plus cviček von mir.« (21. 5. 1934, Cvetko 1988, S. 290).

Oder, er schreibt vor der Ankunft des Osterc in Prag: „Jetzt gib nur schön acht auf Dich und trinke viel Wasser ohne cviček, damit Du nächstes Jahr unser Bier aushältst.“ (19. 11. 1934, Cvetko 1988, S. 293)

Am 8. Mai 1934 trat Reiner in Ljubljana im Rahmen des Intimkonzertes moderner Musik auf. Das Konzert blieb in der slowenischen kritischen Besinnung wegen der berühmten Worte des Kritikers und Komponisten Lucijan Marija Škerjanc verzeichnet: „Ich habe keine Zweifel: Diese Musik ist bis zur Trommel gekommen und kommt auch bald auf die Trommel." ${ }^{8}$ (Loparnik 1995, S. 26)

Diese Kritik verursachte natürlich große Empörung unter den Freunden und Schülern des Osterc, die scharf und auch spitz und zynisch darauf reagierten. Šturm schrieb aus Prag einen gemeinsamen Brief an Osterc und Reiner:

„Meiner Meinung nach langweilen sich er [Škerjanc] und seine Musiker auf der Trommel und möchten ein wenig lustige und gesunde Gesellschaft bekommen.... [...] Mich wundert es, dass Škerjanc nicht einmal seine Skripten aus der Instrumentenlehre beherrscht. Dort sagt er, dass die Trommel ein rhythmischer Instrument ist und hat bei uns nicht einmal den Rhythmus gefunden, jedoch behauptet er, dass wir auf der Trommel sind!“ (12. 5. 1934, Cvetko 1988: 338)

8 „Auf die Trommel zu kommen“ bedeutet in der slowenischen Sprache das Gleiche wie „Unter den Hammer zu kommen“ auf Deutsch, also bankrottieren. 
Unter anderem dachte Šturm offenkundig an eine »Gegenmaßnahme« mit dem Konzert in Prag, in welchem Škerjanc lächerlich gemacht sein sollte. Jedoch kam es später nicht dazu. Die Streitigkeit verursachte eine scharfe Spaltung mit Škerjanc, die auch in Kritik von Osterc über das Dirigieren von Škerjanc in der Orchesterproduktion des Konservatoriums im November 1934 zu erkennen ist: ,alle Tempi waren von Anfang bis Ende zu langsam, überhaupt keine Dynamik, Intonation, insbesondere bei Blasinstrumenten unmöglich. “ (Loparnik 1995, S. 26)

Reiner nennt in einem von den Briefen an Osterc später Lucijan Marija Škerjanc gar „Luzifer Maria“ (13. 5. 1934, Cvetko 1988, S. 289).

In den Briefen fühlt man auch ein Echo auf die immer schwieriger werdenden politischen Verhältnisse, bereits 1934 schreibt er über die Gefahr einer Hitler Revolution in Wien wie folgt:

„In Wien gibt es wie gewöhnlich großes Gejammer. Scheinbar wird es doch bald Hitlerrevolution dort geben. [...] Sonst sieht es, wie gesagt, in Wien sehr schlecht aus und heute sind damit keine großen Zukunftspläne zu machen.« (21. 5. 1934, Cvetko 1988, S. 290)

Nicht zuletzt führte möglicherweise eben die lähmende Atmosphäre nach deutscher Besatzung der Tschechoslowakei im Jahre 1939 zur allmählichen Einstellung des Briefwechsels zwischen Reiner und Osterc. Ihre Korrespondenz war in den Jahren 1933-1935 höchst intensiv, danach schrumpfte ihr einst wöchentlicher Briefwechsel, so dass zwischen dem 2. 10. 1935 und dem 8. 11. 1936, also mehr als ein Jahr, kein Brief vorliegt (in der Zwischenzeit war Osterc zwar im April 1936 mit dem Trboveljski slavček Chor in Prag). Im Mai 1937 schreibt Reiner noch einen kurzen Brief, den er wie folgt beginnt:

„Du wirst natürlich sagen, dass ich ein altes Schwein bin, dass ich nicht schreibe, dafür tue ich es aber heute, ohne etwas von Dir zu wollen, d. h. aus L'art pour l'artismus.“" (20. 5. 1937, Cvetko 1988, S. 301).

Der letzte Brief, vermutlich aus dem Jahr 1938, ist in der tschechischen Sprache verfasst und schließt ihren reichen Briefwechsel in einer herben Weise wie folgt ab: „Já jsem momentalněv situaci labilně a napiši Ti podrobneji, až bude všechno v pořadku. “(12. 8. 1938?, Cvetko 1988, S. 301)

Er fühlte sich bereits einiger Zeit immer mehr weggestoßen. Zu Weinachten 1936 klagt er Osterc sein Leid: „Ich habe ja jetzt vielfache Opposition an mir hängen: als Hába-Schüler, als 'Rytmiker', als Burianist und zuletzt auch als 'ich'." (Cvetko 1988, S. 300)

Der Gesundheitszustand von Osterc verschlechterte sich innerhalb der nächsten Zeit sehr schnell, was zu seinem zu früherem Tod führte. Reiner hatte aber eine unverständliche Verfolgung zu überstehen, erstens als jüdischer „Ich“, und später als „Hába-Schüler“ und „Formalist“, worunter in den geänderten politischen Verhältnissen ein „Instrument in Händen der Bourgeoisie, gegen dem programmatischen Ideenfortschritt" zu verstehen war, wie es der Parteiapparatschick Barvík bezeichnete (Svatos 2010, S. 12). Dieser kommentierte die Reiner Musik mit den Worten: „Ist das wirklich ein Fortschritt? Ist das Kakopho- 
nie - im Vergleich zu Smetanas Freude und Optimismus - eine Art des Fortschritts, welche wir womöglich als die Musik der neuen Zeit verkündigen können?" (Svatos 2010, S. 14)

Trotz des schwierigen persönlichen Schicksals beider Musiker, Freunde, bleiben Reiner und Osterc ein Symbol enger Kontakte zwischen der tschechischen und der slowenischen Musik, Kontakte, die nicht zuletzt zur Bildung unserer zeitgenössischen Kultur produktiv beigebracht haben.

\section{Bibliography}

BEDINA, Katarina. List nove glasbe: Osebnost in delo Frana Šturma. Ljubljana: Cankarjeva založba, 1981.

BEDINA, Katarina (Hrsg.). Zbornik ponatisov o življenju in delu Slavka Osterca. Ob stoletnici skladateljevega rojstva. Ljubljana: Oddelek za muzikologijo Filozofske fakultete v Ljubljani in Slovensko muzikološko društvo (Varia musicologica 2), 1995.

CVETKO, Dragotin. Fragment glasbene moderne, iz pisem Slavku Ostercu. Ljubljana: Slovenska akademija znanosti in umetnosti (Viri za zgodovino Slovencev 11), 1988.

CVETKO, Dragotin. Osebnost Slavka Osterca. Ljubljana: Cankarjeva založba, 1993.

KURET, Primož (Hrsg.). Glasba med obema vojnama in Slavko Osterc: zbornik predavanj. Ljubljana: Festival (Slovenski glasbeni dnevi 1995), 1996.

LOPARNIK, Borut. Biti skladatelj: pogovori s Primožem Ramovšem, Ljubljana: Slovenska matica, 1984.

LOPARNIK, Borut (Hrsg.). Moja smer je skrajna levica: razstava ob stoletnici rojstva Slavka Osterca, Ljubljana, Kulturno-informacijski center Križanke, 11.-28. oktober 1995. Ljubljana: Oddelek za muzikologijo Filozofske fakultete, 1995.

POKORN, Danilo. Slavko Osterc (ob sedemdesetletnici njegovega rojstva). Ljubljana: Prosvetni servis, 1965.

REITTEREROVÁ, Vlasta - REITTERER, Hubert. Alois Hába - Slavko Osterc: Briefwechsel 19311940. In: Musikgeschichte in Mittel- und Osteuropa (Heft 10), Helmut Loos (Hrsg.), 2005, S. 160-172.

RIJAVEC, Andrej. Kompozicijski stavek komornih instrumentalnih del Slavka Osterca. Ljubljana: Slovenska akademija znanosti in umetnosti (Dissertationes VII/4), 1972.

ŠIFTAR, Vanek (Hrsg.). Slavko Osterc. Murska Sobota: Pomurska založba, 1963.

SPURNÝ, Lubomír. The Hába School, Musicologica Brunensia 46/1-2, 2011, S. 141-148.

STEFANIJA, Leon. Osterc in Hába. Muzikološki zbornik 31, 1995, S. 33-41.

SVATOS, Thomas D. Sovietizing Czechoslovak Music: The "Hatchet-Man" Miroslav Barvík and his Speech "The Composers Go with the People". Music Eீ Politics 4/1 (Winter 2010). http:// dx.doi.org/10.3998/mp.9460447.0004.101.

TIBAUT, Ema (Hrsg.). Spomini še živijo. Ob 100-letnici rojstva skladatelja Slavka Osterca. Ljutomer: Zveza kulturnih organizacij Ljutomer, 1995.

TYRELL, John. Janáček: Years of a Life Volume 2 (1914-1928): Tsar of the Forests, London: Faber and Faber, 2011.

WEISS, Jernej. Emerik Beran (1868-1940): samotni svetovljan. Maribor: Litera, 2008.

This work can be used in accordance with the Creative Commons BY-SA 4.0 International license terms and conditions (https://creativecommons.org/licenses/by-sa/4.0/legalcode). This does not apply to works or elements (such as image or photographs) that are used in the work under a contractual license or exception or limitation to relevant rights. 
